\title{
Kniegelenksnahe Frakturen bei Knie-TEP
}

\author{
Karl-Heinz Frosch, Jürgen Madert
}

\section{Zusammenfassung}

Durch die zunehmende Endoprothetisierung der Bevölkerung nimmt auch die Anzahl an periprothetischen Frakturen insbesondere des Kniegelenks zu. Ca. 95\% aller periprothetischen Frakturen betreffen das distale Femur, etwa $4 \%$ die proximale Tibia. Hauptrisikofaktoren, eine periprothetische Fraktur nach Knieendoprothese zu erleiden, sind aktive Patienten unter 60 Jahren, stattgehabter Endoprothesenwechsel, Osteoporose und, sofern es die periprothetischen Frakturen der Patella betrifft, ein femorales Overstuffing, ein verkürztes Lig. patellae und die Durchführung eines Lateral Releases. Am Femur haben sich als Osteosyntheseverfahren die winkelstabile Plattenosteosynthese oder auch der retrograde Marknagel, welcher biomechanische Vorteile besitzt, durchgesetzt. Drahtcerclagen können die mechanische Stabilität der Osteosynthese erhöhen. Bei gelockerten Implantaten ist i.d.R. der Wechsel auf ein Revisionsimplantat notwendig. $\mathrm{Zu}$ beachten ist, dass nach aktueller Studienlage die meisten periprothetischen Frakturen der Tibia mit einem gelockerten Implantat einhergehen, femoral sind die Komponenten meist fest integriert. Periprothetische Frakturen der Patella können zu erheblichen Problemen mit verzögerter Heilung aufgrund der meist problematischen Blutversorgung führen, was bereits bei der Primärimplantation zu berücksichtigen ist. Aufgrund hoher Komplikationsraten von periprothetischen Frakturen am Kniegelenk, die zwischen 12 und $41 \%$ angegeben werden, ist eine optimale Versorgung essenziell.

\section{Periprosthetic Fractures around the Knee}

With the increasing number of arthroplasties being performed, an elevated incidence of periprosthetic fractures around the knee can be observed. About $95 \%$ of the fractures are located at the distal femur, $4 \%$ are found at the tibia and $1 \%$ at the patella. The main risk factors to suffer from a peripros- thetic fracture around the knee are high activity levels combined with an age lower than 60 years, revisionarthroplasty, long stems and osteoporosis. Femoral overstuffing, patella baja and lateral release are risk factors for patella fractures. At the femoral location, angle stable plates or a retrograde nail are the prefered implants. Cerclage wires enhance the biomechanical stability. Loose implants result in the necessity to perform a revision arthroplasty. According to the current literature, most periprosthetic fractures at the tibial side are accompanied with a loose implant, at the femoral side the implants are stably integrated in most cases. Periprosthetic patella fractures often have healing problems due to an impaired blood supply after an anteromedial arthrotomy. In general there is a high complication rate of 12 to $41 \%$ in the treatment of periprosthetic fractures around the knee. This should be considered for the primary knee arthroplasty as well as in the treatment of the periprosthetic fractures.

\section{Einleitung}

Die Anzahl an Endoprothesenimplantationen in Deutschland hat in den letzten Jahren deutlich zugenommen. Mittlerweile werden ca. 170000 Knieprothesen in Deutschland implantiert und ca. 10000 Wechseloperationen durchgeführt. Die Rate an periprothetischen Frakturen nach Knietotalendoprothesenimplantation beträgt $0,6-2,5 \%$. Betroffen ist meist das distale Femur. In knapp 40\% der Fälle treten periprothetische Frakturen am Kniegelenk bei vor-

OP-JOURNAL 2015; 31: 37-44

(C) Georg Thieme Verlag KG Stuttgart · New York DOI http://dx.doi.org/10.1055/s-0035-1545838 handenen Revisionsendoprothesen auf $[1,25,44]$.

Insgesamt handelt es sich bei einer periprothetischen Fraktur am Kniegelenk um eine schwere Verletzung. Sowohl die konservative als auch die operative Therapie sind von hohen Komplikationsraten begleitet.

Die Komplikationsrate nach operativer Versorgung einer periprothetischen Fraktur nach Knietotalendoprothese beträgt bis zu $41 \%$ [34]. In 29\% der Fälle wird aufgrund von Komplikationen eine operative Revision nach primärer Osteosynthese durchgeführt [34].
Risikofaktoren, eine periprothetische Fraktur am Kniegelenk zu erleiden, wurden von Singh et al. bei 21723 Knieprothesen identifiziert [40]. Die postoperative kumulative Inzidenz nach Primärendoprothesenimplantation betrug $1,1 \%$, nach Wechselendoprothesenimplantation 2,5\%. Letztere stellte einen der Hauptrisikofaktoren für das Auftreten einer periprothetischen Fraktur dar. Das Risiko, eine periprothetische Fraktur zu erleiden, war in der Altersgruppe zwischen 61 und 70 Jahren halb so hoch wie bei Patienten unter 60 Jahren und war bei über 80-Jährigen nicht wesentlich erhöht. Patienten im Alter zwischen 71 und 80 Jahren hatten ebenfalls kein wesentlich erhöhtes Risiko [40]. Nicht zu unterschätzen sind nach unserer Erfah- 


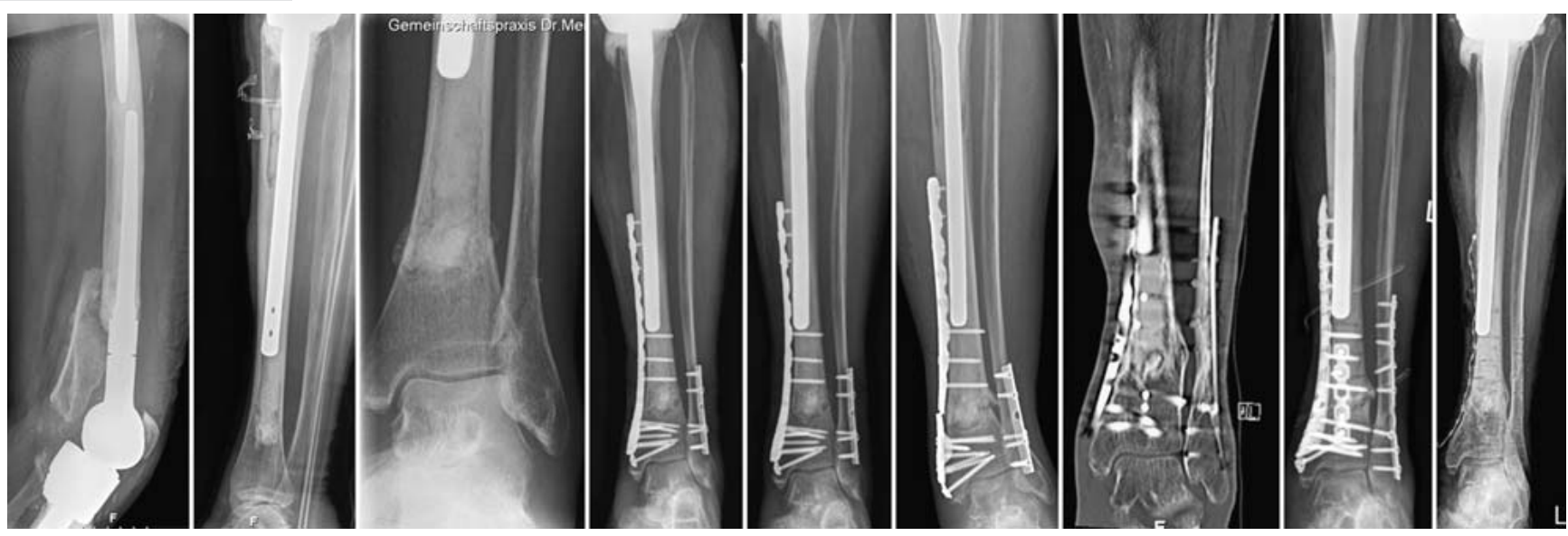

Abb. 1 Periprothetische Ermüdungsfraktur der distalen Tibia bei überlangem Stem und zu langem Zementzapfen, der noch mehr als $4 \mathrm{~cm}$ über die Spitze des Stems nach distal reicht.

Tab. 1 Klassifikation der Femurfraktur bei periprothetischer Fraktur bei Knieendoprothese nach Rorabeck [33].

\section{femorale Komponente:}

Rorabeck I: nicht dislozierte Frakturen $\left(<5 \mathrm{~mm}\right.$ und $\left.<5^{\circ}\right)$ bei stabil integriertem Implantat

Rorabeck II: stabile Prothese bei dislozierter Fraktur

Rorabeck III: Fraktur um eine gelockerte Prothese

rung auch lange und insbesondere zementierte Stems, die erhebliche Spannungspitzen am Ende der Stems verursachen können und nicht selten $\mathrm{zu}$ Ermüdungsfrakturen führen. Die Therapie solcher Ermüdungsfrakturen kann sich als sehr hartnäckig und schwierig erweisen (Abb. 1).

Ein hoher Body-Mass-Index, das vorliegen einer Herzerkrankung, tiefe Beinvenenthrombose wie auch zementierte oder zementfreie Technik stellten keine Risikofaktoren dar. Patienten mit ASA 1 erlitten doppelt so viele periprothetische Frakturen wie Patienten in der Gruppe ASA 2-4. Das höchste Risiko, eine periprothetische Fraktur nach Knieendoprothesenimplantation zu erleiden, haben jüngere Patienten, die entweder körperlich sehr aktiv sind oder aufgrund von entzündlichen Gelenkerkrankungen mittels Steroiden behandelt wurden [40]. Als weiterer Risikofaktor für eine periprothetische Fraktur wurde auch das Vorliegen einer manifesten Osteoporose identifiziert [5].

Mehrere Arbeitsgruppen konnten nachweisen, dass ein femorales Notching Grad 1 oder 2 nach Tayside zu keiner er- höhten Rate an periprothetischen Frakturen führt $[17,32]$. Mit „femoralem Notching" ist dabei das Unterschneiden bzw. die Arrosion der ventralen Femurkortikalis durch einen zu weit dorsal gelegenen ventralen Sägeschnitt für die Knieendoprothese gemeint. Dabei zeigte sich in einer Serie von 200 Patienten mit Knieendoprothese in $41 \%$ der Fälle ein ventrales Notching [17]. Innerhalb von 9 Jahren kam es zu insgesamt 3 periprothetischen Frakturen, die alle durch Sturz auf das Knie erfolgten. Aus der Gruppe der Patienten mit femoralem Notching erlitt nur ein Patient mit einem Notching Grad 2 nach Tayside eine periprothetische Fraktur. Ähnliche Ergebnisse werden auch von Ritter et al. beschrieben, die bei 1089 Knieprothesen in 29,8\% der Fälle ein femorales Notching beobachten konnten, welches jedoch weder mit einer erhöhten Frakturrate noch mit einem schlechteren Outcome assoziiert war [32]. In einer Finite-Elemente-Analyse konnte gezeigt werden, dass bei mehr als $3 \mathrm{~mm}$ Notching im Bereich der ventralen Kortikalis des distalen Femurs mit kantigem Kalibersprung direkt am proximalen Ende der Prothese eine erhebliche Stresskonzentration entstehen kann, woraus ein erhöhtes Frakturrisiko resultieren kann [46].

\section{Klassifikation}

Für die periprothetischen Frakturen bei liegender Knieprothese werden im Wesentlichen 3 Klassifikationen verwendet: diejenige für die femorale nach Rorabeck [33], diejenige für die tibiale Komponente nach Felix [14] und diejenige für periprothetische Patellafrakturen nach Keating [22]. Andere Klassifikationen, die v.a. die knöcherne Qualität berücksichtigen, haben sich nicht bewährt [24,34]. Die Rora-
beck-Klassifikation [33] berücksichtigt stabile und gelockerte Implantate sowie den Grad der Dislokation (Tab. 1).

Die Rorabeck-Klassifikation hat den Vorteil, dass sie sehr einfach ist. Nachteil ist jedoch, dass der Charakter der Fraktur selbst kaum berücksichtigt wird und die Einteilung nur sehr grob ist. Ein Behandlungsalgorithmus ist aus der RorabeckKlassifikation nur schwer abzuleiten. Ruchholz et al. empfehlen deshalb, zusätzlich die AO-Klassifikation für distale Femurfrakturen zu verwenden [34].

Die Tibiafraktur wird am häufigsten nach der Klassifikation von Felix eingeteilt, die auf einer Studie mit 102 Patienten beruht [14]. Es werden nach Lokalisation der Fraktur 4 Typen unterschieden, die jeweils noch zusätzlich mit einem Buchstaben versehen werden, der beschreibt, ob die Prothese locker oder stabil ist. Die Felix-Klassifikation lässt einen strukturierten Behandlungsalgorithmus zu.

Für die periprothetischen Patellafrakturen existieren insgesamt 3 Klassifikationen, die insgesamt jedoch relativ wenig gebräuchlich sind [2]. Auch werden mit keiner der 3 Klassifikationen die Charakteristik der Fraktur, der Frakturverlauf oder die zugrunde liegende Pathologie erfasst [2]. Als die am ehesten brauchbare Klassifikation erscheint diejenige von Keating et al. [22].

\section{Diagnostik}

Bei periprothetischen Frakturen ist eine detaillierte Anamnese von Bedeutung. Insbesondere ist wichtig zu erfragen, wie lange das Implantat bereits vorhanden ist, welche operativen Eingriffe und Revisionen durchgeführt wurden und 


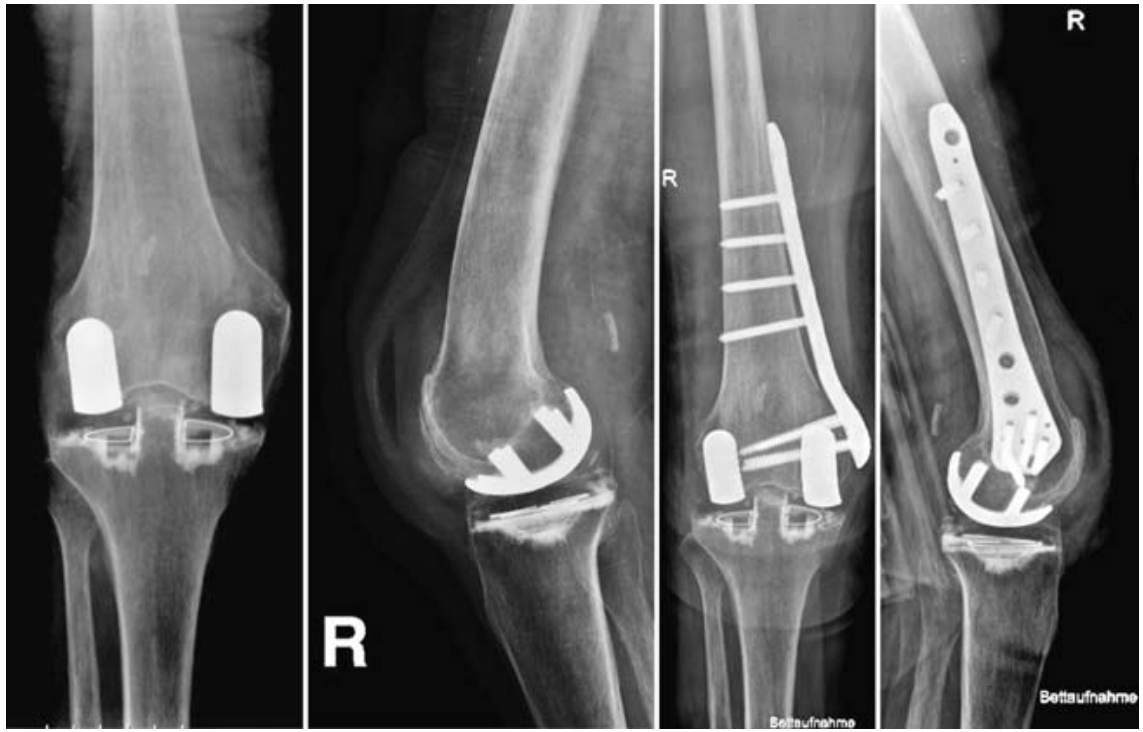

Abb. 2 88-jährige Patientin in reduziertem Allgemeinzustand nach Sturz auf das rechte Knie. Fraktur der medialen Kondyle nach bikondylärer Schlittenprothese und Patellektomie vor mehr als 10 Jahren. Geschlossene Reposition und Versorgung mittels eingeschobener, winkelstabiler Plattenosteosynthese.

ob bereits Schmerzen und Beschwerden vor dem Frakturereignis bestanden. Oft sind einfache Röntgenaufnahmen in 2 Ebenen ausreichend [34], insbesondere bei implantatfernen Frakturen (z.B. Felix Typ III). Bei Frakturen, die direkt in das Implantat einstrahlen, halten wir eine CT-Untersuchung für hilfreich und führen diese regelhaft durch. Dabei kann der Frakturverlauf genau evaluiert und versteckte Fissuren zur Darstellung gebracht werden. Insbesondere bei der Planung minimalinvasiver Techniken kann das CT die Operation vereinfachen.

Die Entscheidung, ob ein Implantat locker oder fest sitzt, kann z.T. schwierig sein. Hier können ältere Röntgenbilder mit der Entwicklung typischer Lysesäume um das Implantat, der Ausbildung von Osteolysen sowie eventueller Implantatsinterung im Verlauf hinweisend sein. Auch die Durchführung einer Computertomografie kann diesbezüglich hilfreich sein. Bei bleibenden Unklarheiten empfehlen wir eher das offene operative Vorgehen und die zusätzliche manuelle Prüfung der Festigkeit der Komponenten. Bei länger bestehenden Implantaten mit verbundenen Schmerzen und Beschwerden über einen längeren Zeitraum stellen wir die Indikation zum Implantatwechsel in Abhängigkeit vom Frakturtyp eher großzügig.

Nicht zu vergessen sind „low-grade“-Infekte, die auch bei einer periprothetischen Fraktur vorliegen können. Bei Verdacht ist hier präoperativ eine Flüssig- keitsaspiration zur bakteriologischen Untersuchung anzustreben. Grundsätzlich entnehmen wir intraoperativ Gewebeproben (keine Watteabstriche) für die bakteriologische und histologische Untersuchung, um einen Infekt auszuschließen.

\section{Konservative oder operative Therapie}

Die konservative Therapie hat zwar ein reduziertes Infektionsrisiko, ist aber meist von langer Immobilisation begleitet [9] und wird deshalb nur in bestimmten Ausnahmefällen empfohlen. In 15 von 30 Fällen konnte nach konservativer Therapie eine deutliche Bewegungseinschränkung festgestellt werden [11]. Über schlechte Ergebnisse wird auch von anderen Autoren berichtet, bei denen viele Patienten nach konservativer Therapie auch im Verlauf noch eine operative Intervention benötigten [20]. Neuere Studien empfehlen deshalb für die Mehrzahl der Fälle die operative Versorgung $[18,34]$. Prospektiv randomisierte Studien hierzu existieren nicht. Auch bei nicht dislozierten Frakturen wird aufgrund der langen Immobilisationsdauer und des hohen Risikos der sekundären Dislokation unter konservativer Therapie die operative Versorgung empfohlen [21, 34]. Aus unserer Sicht ist die konservative Therapie Patienten mit nicht oder gering dislozierten Frakturen und erheblichen operativen Risiken aufgrund medizinischer Vorerkrankungen (z.B. ASA 3 oder 4) oder kritischen Weichteilen (z.B. Schuppenflechte etc.) vorbehalten.

\section{Periprothetische Fraktur des distalen Femurs}

Mit konventionellen Plattensystemen werden Komplikationsraten bei periprothetischen Frakturen des distalen Femurs mit bis zu 51\% angegeben [21]. Deutlich bessere Ergebnisse werden in neueren Studien mittels winkelstabiler Plattensysteme beobachtet (Abb. 2). Vergleichsstudien konnten zeigen, dass winkelstabile Platten hier deutliche Vorteile gegenüber konventionellen Platten haben [4]. Dabei heilten 13 von 14 mit winkelstabilen Platten versorgte Frakturen komplikationslos, bei nicht winkelstabilen Platten war in 7 von 19 Fällen eine operative Revision nötig [4]. Auch sekundäre Achsenabweichungen und Korrekturverlust waren signifikant häufiger bei nicht winkelstabilen Implantaten [4]. Interessanterweise wird auch mit der $95^{\circ}$-Klingenplatte über gute $\mathrm{Er}$ gebnisse mit wenig Korrekturverlust und niedrigen Revisionsraten berichtet [43]. Dabei war die Revisionsrate mit der Klingenplatte $6 \%$, diejenige mit der winkelstabilen LCP (Locking Compression Plate) $43 \%$ [43]. Hier ist allerdings anzumerken, dass in keiner anderen Studie mit winkelstabilen Implantaten so hohe Komplikationsraten angegeben werden. Darüber hinaus sind andere Autoren der Meinung, dass die klinischen Ergebnisse durch polyaxiale winkelstabile Implantate nochmals verbessert werden können [34]. Ein TEP-Wechsel ist bei gelockertem Implantat notwendig (Abb. 3)

\section{Retrograder Nagel oder Platte?}

In aktuellen Arbeiten konnte gezeigt werden, dass der retrograde Nagel biomechanisch vorteilhaft gegenüber der winkelstabilen Platte $\mathrm{zu}$ sein scheint [30]. Dabei war das stabilste Konstrukt der retrograde Nagel mit winkelstabiler Verriegelung, dann der retrograde Nagel mit konventioneller (nicht winkelstabiler) Verriegelung und am wenigsten stabil erwies sich die winkelstabile Platte [30]. Chen et al. hingegen konnten in einer Finite-Elemente-Analyse zeigen, dass der retrograde Nagel wie auch die winkelstabile Platte am distalen Femur ähnliche biomechanische Eigenschaften haben und empfiehlt zur weiteren Erhöhung der Primärstabilität zusätzlich die Verwendung eines intramedullären „allograft rods“ [10]. Sun et al. konnten ebenfalls in einer Finite-Elemente-Analyse zeigen, dass der mechanische Stress auf das Femur mit zunehmender Länge der Stems vor allem von Knieprothesen, 


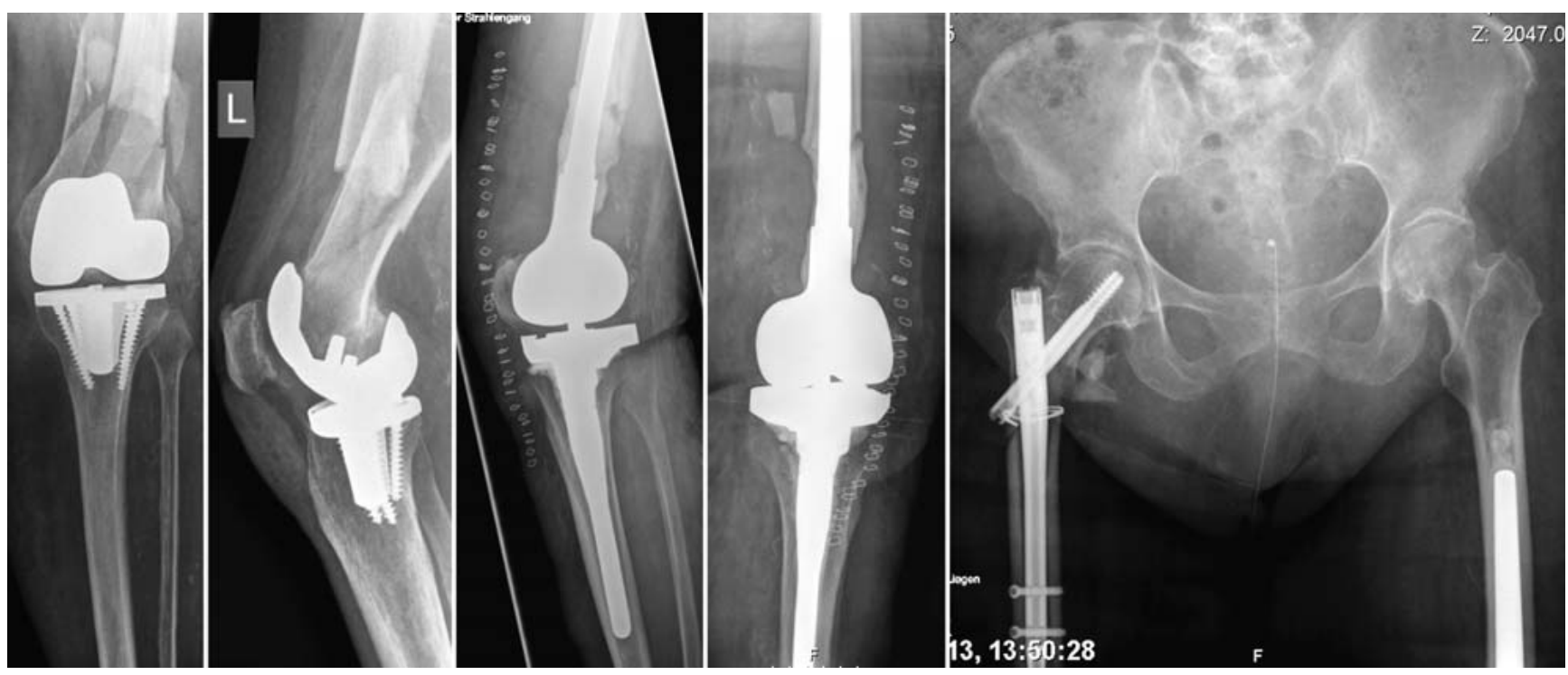

Abb. 3 Periprothetische Femurfraktur Rorabeck II: Die 88-jährige Patientin klagte präoperativ bereits über seit Monaten bestehende, zunehmende Knieschmerzen, sodass letztlich ein gelockertes Implantat nicht ausgeschlossen werden konnte. Aufgrund des Allgemeinzustands hätte die Patientin postoperativ nicht entlasten können. Es wurde ein primärer TEP-Wechsel auf ein gekoppeltes, zementiertes Implantat durchgeführt. Die Patientin konnte bei komplikationslosem postoperativem Verlauf sofort mobilisiert werden.
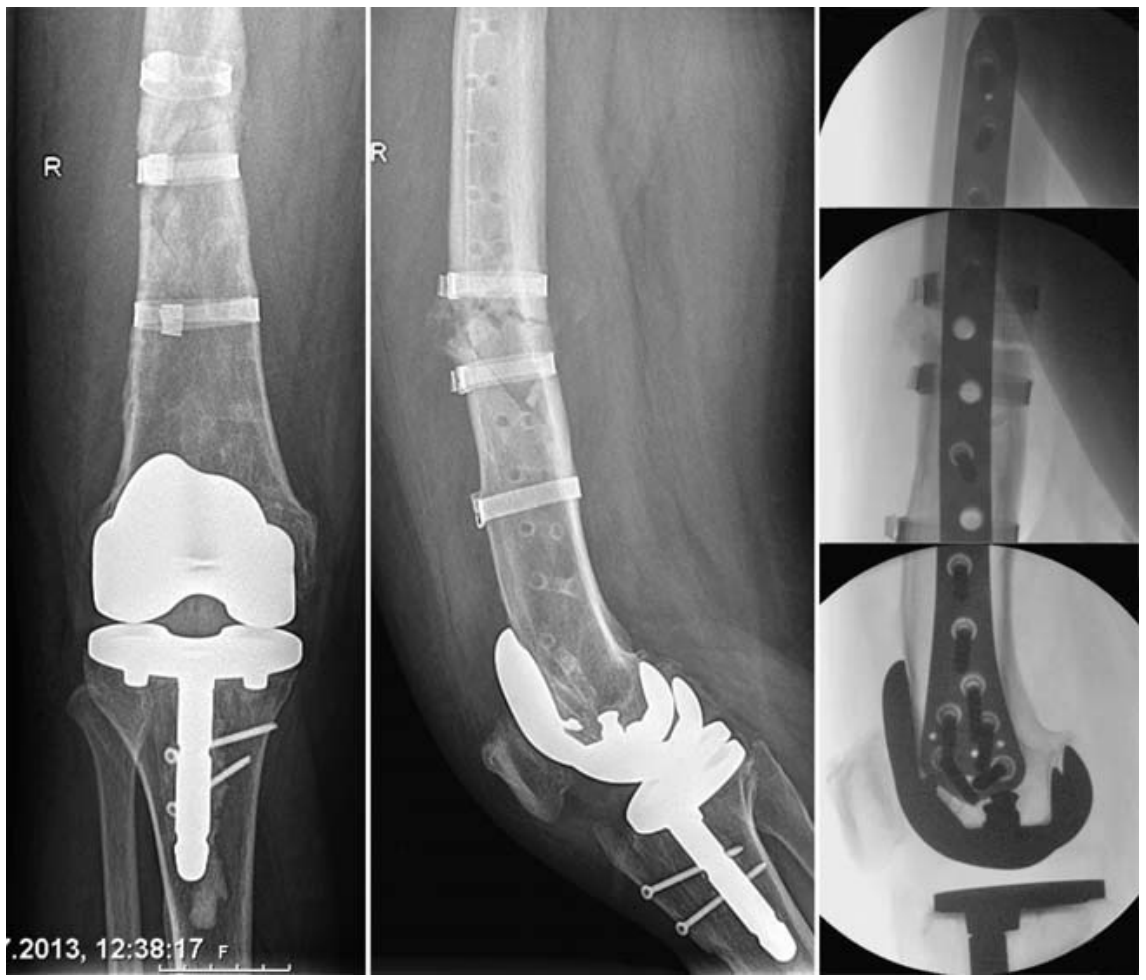

Abb. 4 Periprothetische Ermüdungsfraktur ohne Trauma nach Anlage von 3 Cerclagen. Vor 2 Jahren hatte die Patientin eine periprothetische Spiralfraktur erlitten, das Metall wurde bereits entfernt, die Fraktur war durchbaut. Nun erneute Stabilisierung mit winkelstabiler Platte. Es kam zur Heilung innerhalb weniger Wochen.

aber auch von Hüftprothesen, erheblich zunimmt [41] und das Risiko, eine periprothetische Fraktur zu erleiden, damit steigt. Eine weitere biomechanische Studie zeigt den Einfluss verschiedener Osteosyntheseimplantate und verschiedener Endoprothesen (inkl. Stems) und wie diese sich gegenseitig bei verschiedenen Implantatkombinationen beeinflussen [35]. Es konnte gezeigt werden, dass eine Hüftprothese zu einer deutlichen Schwächung der Stabilität (ca. 33\%) des Femurs führt. Ein retrograder Nagel (oder auch Stiel einer Knieendoprothe- se) bei liegender Hüftprothese führt zur weiteren Schwächung der Stabilität des Femurs durch das hohe Biegemoment der beiden Implantate, welche auf eine nur kurze knöcherne Strecke einwirken. Bei dieser Kombination wird von den Autoren deshalb eine zusätzliche Stabilisierung mittels winkelstabiler Platte empfohlen, um die Strecke zwischen den beiden Implantaten zu überbrücken. Alternativ wird die Verwendung von „Steckhülsen“ empfohlen, welche die beiden Implantate miteinander verbinden und damit zu einer hohen mechanischen Stabilität führen.

In den letzten 12 Monaten sind auch mehrere klinische Vergleichsstudien zum Thema winkelstabile Platte vs. retrograder Marknagel erschienen [3,16,23,28]. Dabei konnten mehrere Gruppen in ihren Vergleichsstudien zwischen retrogradem Nagel vs. winkelstabiler Platte bei periprothetischen Frakturen im Bereich des Kniegelenks keine Unterschiede bez. Heilungs- und Komplikationsrate feststellen $[3,16,23]$. Gondalia et al. fanden Komplikationsraten zwischen 27 und 29\% in beiden Gruppen, dabei konnte bei der winkelstabilen Platte eine höhere „nonunion“-Rate gefunden werden, beim retrograden Nagel wurden hingegen mehr Refrakturen beobachtet [16]. Die Komplikationsrate war dabei ausschließlich vom Alter der Patienten abhängig [16].

Obwohl der retrograde Marknagel biomechanisch eher leicht vorteilhaft gegenüber der Plattenosteosynthese er- 
scheint, existiert in der aktuellen Literatur nur 1 nennenswerte Publikation, die klinisch deutliche Vorteile der intramedullären Stabilisierung zeigt [28]. Bei 95 periprothetischen Frakturen bei liegender Knieendoprothese kam es unter retrograder Marknagelung zu „non-unions/ delayed unions“ in 9\% der Fälle, mit der winkelstabilen Platte hingegen bei $19 \%$ der Fälle [28]. Obwohl im Durchschnitt beim retrograden Nagel nur 3,8 und bei der winkelstabilen Platte 5,0 winkelstabile Schrauben distal verwendet wurden, lag die Komplikationsrate bei der Platte doppelt so hoch [28].

$\mathrm{Zu}$ beachten ist, dass eine retrograde Marknagelung des Femurs bei fast allen bikondylären Oberflächenersatzprothesen möglich ist. Es sollte dennoch im Einzelfall geprüft werden, ob der Marknagel in der interkondylären Notch des jeweiligen Implantats Platz findet. Bei „posterior-stabilized“-Implantaten ist eine retrograde Marknagelung nicht möglich, auch nicht bei Implantaten mit höheren Kopplungsgraden. Hier sollte jeweils eine winkelstabile Platte Verwendung finden.

\section{Minimalinvasiv oder offen?}

Gerade nach Implantation einer Knieprothese, welche i.d.R. über einen ventralen Zugang erfolgt, ist die Zugangswahl für die Versorgung einer periprothetischen Fraktur von großer Bedeutung. Über den ehemaligen ventralen Zugang ist die Frakturversorgung meist nur mittels Marknagelung möglich. Bei einer geplanten Plattenosteosynthese ist i.d. R. ein weiterer lateraler oder medialer $\mathrm{Zu}-$ gang notwendig (Abb.2). Um die Blutversorgung möglichst wenig zu kompromittieren und Wundheilungsstörungen zu vermeiden, verwenden viele Autoren einen minimalinvasiven Zugang $[13,15$, 45]. Laut Ruchholz werden bei minimalinvasiven Zugängen niedrigere Revisionsraten $(0-12,5 \%)$ beobachtet als mit offenen Versorgungen (7-29\%) [34]. Eine direkte Vergleichbarkeit der Studien ist jedoch aufgrund der unterschiedlichen Patientengruppen und Frakturtypen nicht möglich [34]. So berichten Ha et al. über niedrige Komplikationsraten und keiner Bewegungseinschränkung nach minimalinvasiver Versorgung von periprothetischen Frakturen des Kniegelenks bei insgesamt 14 Patienten [18]. Prospektiv randomisierte Studien oder Studien mit höherem Evidenzlevel zum Thema minimalinvasiv vs. offen sind derzeit noch nicht vorhanden.
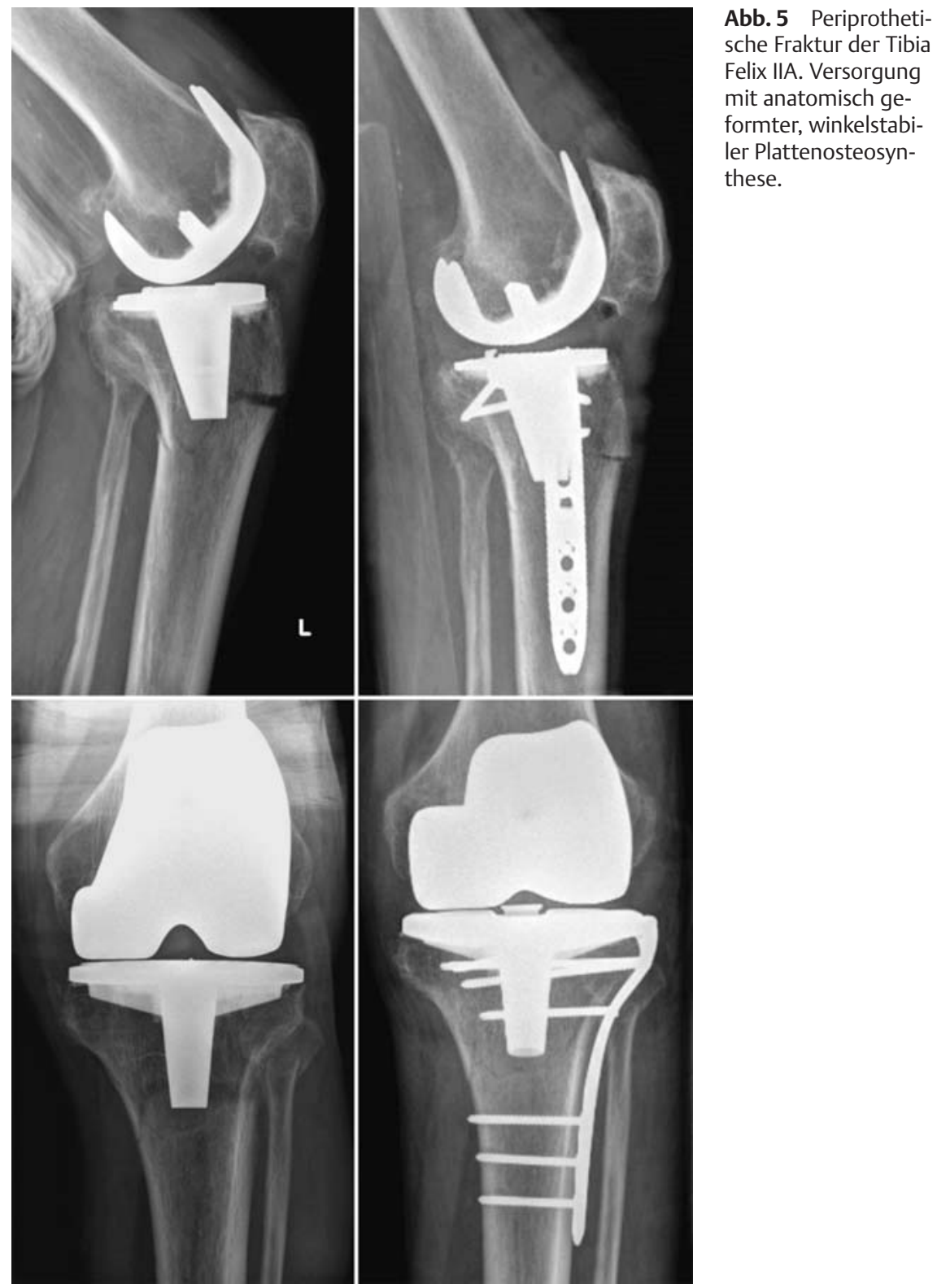
cerclagen niedrigere Revisionsraten $(0 \%)$ hatten als Patienten ohne Drahtcerclage $(20,8 \%)$ bei jeweiliger Verwendung winkelstabiler Platten [12]. Wie viele Cerclagen bei einer periprothetischen Fraktur verwendet werden können oder sollen, ist jedoch letztlich nicht geklärt. Da die periostale Blutversorgung im Schaftbereich längs verläuft und von multiplen Gefäßen sowohl proximal auch als distal versorgt wird, dürfte die Anlage einer

Drahtcerclage biologisch wenig nachteilig sein. Dies bestätigen auch die guten klinischen Ergebnisse [12]. Mehrere Cerclagen im Schaftbereich an mehreren Lokalisationen halten wir jedoch nicht für sinnvoll, was letztlich auch das Fallbeispiel demonstriert (Abb.4).

\section{Periprothetische Fraktur der Patella}

Läsionen des Streckapparats treten in Form von periprothetischen Patellafrakturen sowie von Quadrizeps- und Patellarsehnenrupturen auf. Die Inzidenz von Rupturen des Streckapparats nach Knieendoprothese liegt in der aktuellen Literatur bei $0,17-2,5 \%$ [7]. Von dieser ohnehin geringen Anzahl betrifft nur ein kleiner Teil periprothetische Frakturen der Patella (Tab. 3). Die Prävalenz einer peri- 


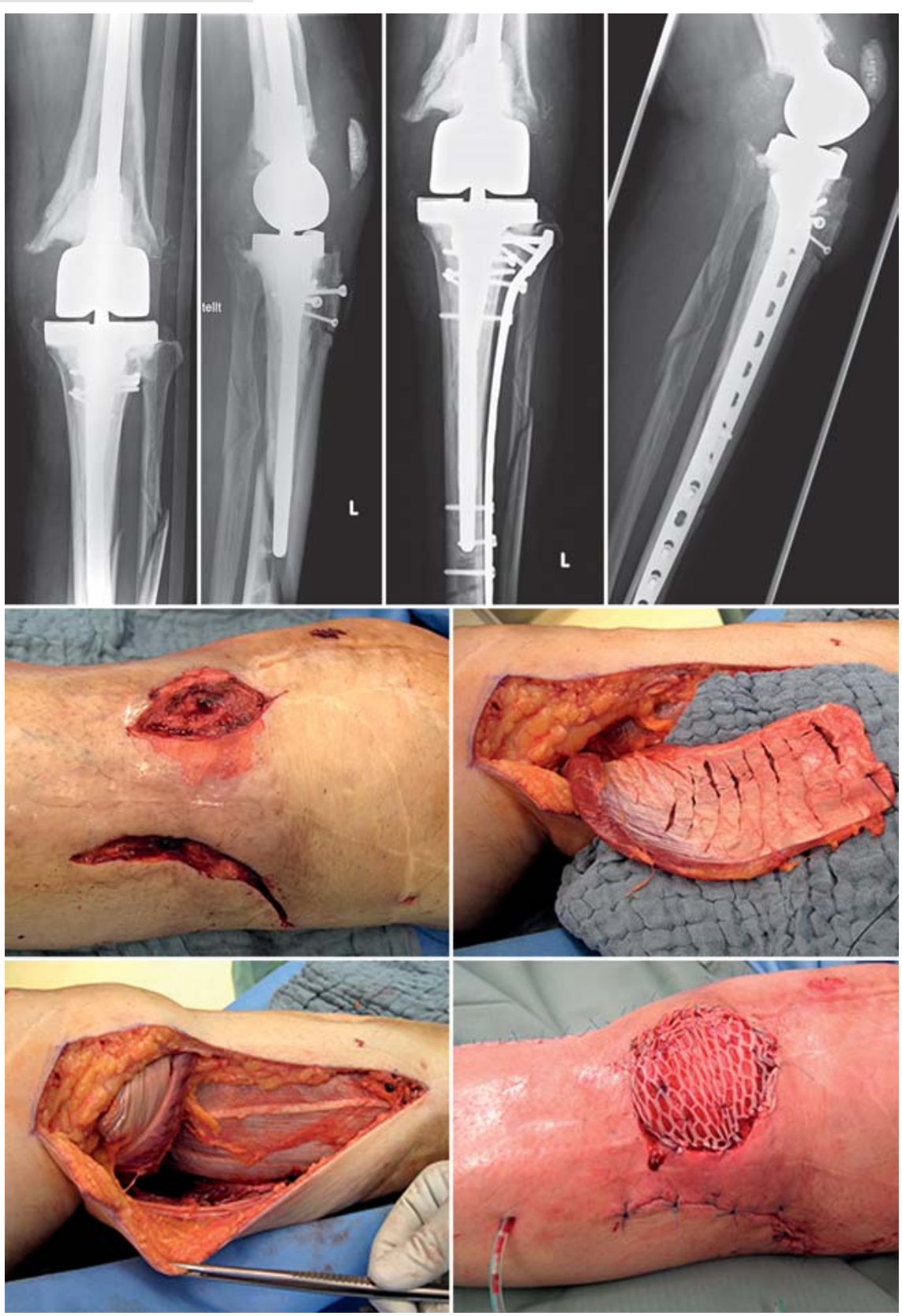

Abb. 6 Periprothetische Spiralfraktur der Tibia nach gekoppelter Knierevisionsendoprothese Typ IIA nach Felix. Durch direkten Sturz auf das Knie findet sich am anteromedialen Tibiakopf ein drittgradiger Weichteilschaden, welcher gleich bei der primären Osteosynthese mittels Gastrocnemiuslappenplastik und Spalthauttransplantation versorgt wird. Die winkelstabile Platte wird eingeschoben, die Fraktur an der Platte reponiert und die Schrauben distal über Stichinzision minimalinvasiv eingebracht. Die Fraktur heilt nach wenigen Wochen ohne weitere Komplikationen aus.

prothetischen Patellafraktur wurde in einer Studie mit 12464 Knieprothesen mit $0,68 \%$ angegeben [29]. Die meisten periprothetischen Patellafrakturen entstehen im Rahmen eines geringgradigen Traumas, Sturz auf das Knie oder exzentrischer Quadrizepskontraktur [2]. Viele werden auch spontan beobachtet [39].

Von wichtiger Bedeutung für das Verständnis periprothetischer Patellafrakturen ist die Blutversorgung der Patella.
Diese kommt v.a. von distal über den inferioren Pol [36]. Insbesondere der proximale Patellapol wird fast ausschließlich über intraossäre Blutgefäße des inferioren Pols versorgt. So konnte gezeigt werden, dass es bei $11 \%$ aller Patellafrakturen zu einer teilweisen oder kompletten Nekrose des proximalen Patellapols kommt bzw. evtl. auftretende Nekrosen betreffen ausschließlich den proximalen Pol [36]. Im Rahmen der Knieendoprothetik konnte szintigrafisch gezeigt wer- den, dass bei ca. $15 \%$ aller Endoprothesen am Knie eine relevante und meist gravierende Störung der Blutversorgung der Patella vorliegt [37]. Bei zusätzlicher Durchführung eines lateralen Releases steigt die Rate auf 56,4\% [37]. Das Auftreten von periprothetischen Patellafrakturen ist deshalb nach lateralem Release und einem Body-Mass-Index $>30 \mathrm{~kg} / \mathrm{m}^{2}$ deutlich erhöht [27].

Auch die Patelladicke spielt als Prädiktor für eine periprothetische Patellafraktur eine Rolle. Eine Dicke der knöchernen Patella unter $15 \mathrm{~mm}$ zeigt in einer FiniteElemente-Analyse erhebliche Stressbelastungen und stellt somit einen Risikofaktor dar [31].

Die Studie mit der derzeit größten Fallzahl beinhaltet 7866 Knieprothesen mit 88 periprothetischen Patellafrakturen [39]. Das mittlere Alter der Patienten betrug 70 Jahre, die Fraktur trat im Mittel 13,4 Monate nach Implantation auf. Die Inzidenz einer spontanen periprothetischen Patellafraktur war signifikant assoziiert mit vorausgegangenen Knieoperationen, vorbestehenden ausgeprägten Achsenabweichungen, postoperativ verminderter Länge der Patellasehne, Verminderung der Patelladicke nach Resektion und einer Patella baja (verminderter Insall-Salvati-Index) [39].

Auch zeigte sich in der Gruppe der Patienten mit periprothetischer Patellafraktur ein signifikant höherer Unterschied des anterior-posterioren Implantatdurchmessers von präoperativ zu postoperativ als in einer Vergleichsgruppe von Patienten ohne Patellafraktur [39]. Das heißt, dass auch ein femorales "Overstuffing“" in a.-p.-Richtung einen signifikanten Risikofaktor für eine periprothetische Patellafraktur nach Knieendoprothese darstellt [39] und deshalb vermieden werden sollte.

Hinsichtlich des Risikos, eine periprothetische Patellafraktur nach Knieendoprothese zu erleiden, ist ein femorales „Notching“ unter $3 \mathrm{~mm}$ sicherlich weniger problematisch als ein ventrales Überstehen des Implantats oder ein „Overstuffing “ der femoralen Komponente in a.-p.-Richtung.

Biomechanische Studien konnten bei Patellaquerfrakturen zeigen, dass die Osteosynthese mittels winkelstabiler Platte signifikant und um ein vielfaches stabiler ist als die kanülierte Zugschraubenosteosynthese in Kombination mit Drahtcer- 

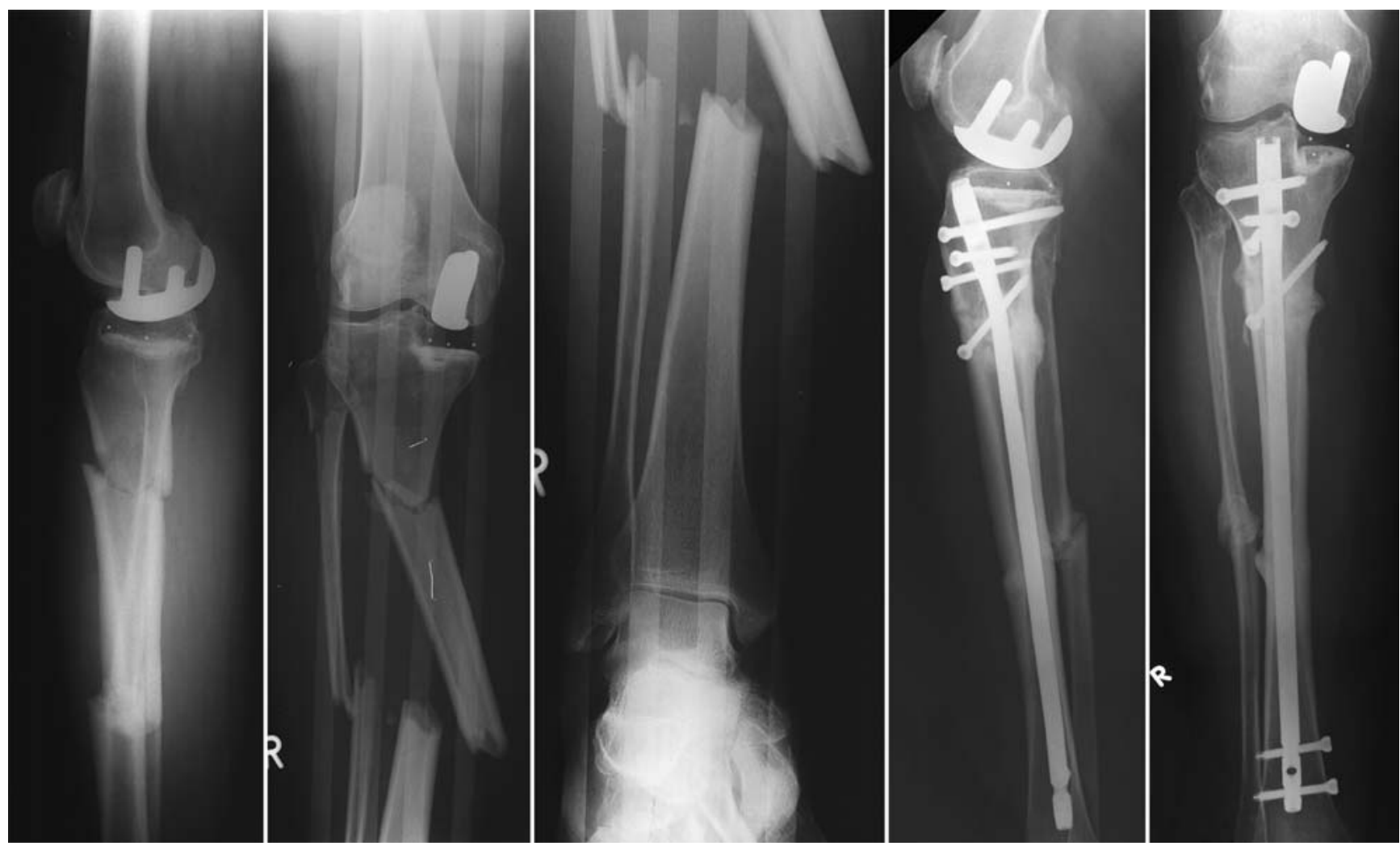

Abb. 7 Periprothetische Fraktur bei liegender Schlittenprothese Felix IIIA. Nach Marknagelung durchbaut die Fraktur innerhalb von 4 Monaten.

clage [42]. Letztere wiederum erwies sich doppelt so stabil wie die klassische Zuggurtungsosteosynthese mit Drähten [42]. Auch andere Autoren wiesen auf die reduzierte biomechanische Qualität und die hohe Komplikationsrate der Zuggurtungsosteosynthese bereits hin, sodass wir diese gerade im Falle einer periprothetischen Patellafraktur nicht empfehlen können. Aufgrund der hohen Komplikationsraten empfehlen wir bei periprothetischen Patellafrakturen die winkelstabile Plattenosteosynthese oder alternativ die kanülierte Zugschraubenosteosynthese mit zusätzlicher Drahtcerclage.

\section{Periprothetische Fraktur der proximalen Tibia}

Nur ca. 4\% der periprothetischen Frakturen nach Knietotalendoprothese betreffen die proximale Tibia [34]. Davon treten ca. $19 \%$ der proximalen Tibiafrakturen bereits intraoperativ während der Prothesenimplantation auf [14]. Eine der wenigen Studien, die in größerem Umfang isoliert auf periprothetische Frakturen der Tibia eingeht, ist diejenige von Felix et al. [14] (Tab. 2). Die Klassifikation von Felix inkl. der sich daraus ergebenden Handlungsempfehlungen ist nach wie vor aktuell. Ca. 80\% der Fraktu-
Tab. 2 Klassifikation der Tibiafraktur bei periprothetischer Fraktur bei Knieendoprothese nach Felix [14].

\section{tibiale Komponente:}

Typ 1: die Fraktur strahlt in den Tibiakopf ein (Fissur oder Spalt)

Typ 2: die Fraktur bezieht den gesamten Tibiakopf auf Höhe des Implantats ein

Typ 3: die Fraktur befindet sich distal der tibialen Komponente

Typ 4: die Fraktur betrifft isoliert die Tuberositas tibiae

Die 4 Frakturtypen werden jeweils noch kombiniert mit dem Zusatz

A - stabile Prothese

B - gelockerte Prothese

C - intraoperative Fraktur

ren gehören zum Typ I oder II. Die meisten periprothetischen Frakturen der Tibia gehen mit einem gelockerten Implantat einher und gehören zum Typ IB oder IIB $[14,19]$. In diesen Fällen ist i.d. R. der Komponentenwechsel, in wenigen Fällen mit zusätzlicher Osteosynthese, notwendig $[14,19]$. Beim Typ IIA und IIIA (Frakturen im Bereich des Implantats bzw. distal davon) empfehlen Felix et al.
Tab.3 Klassifikation periprothetischer Patellafrakturen nach Keating et al. [22].

Patella

Typ 1: vertikal verlaufende Fraktur mit intaktem Streckapparat

\begin{tabular}{l}
\hline Typ 2A: Dislokation $<1 \mathrm{~cm}$ \\
\hline Typ 2B: Dislokation $>1 \mathrm{~cm}$ \\
\hline $\begin{array}{l}\text { Typ 3: Fraktur mit intaktem Streckapparat } \\
\text { und gelockertem Implantat }\end{array}$
\end{tabular}

die Anwendung der allgemeinen Osteosyntheseprinzipien. Dies bedeutet i.d.R. die offene Reposition und Fixation mit winkelstabilen Platten (Abb.5 und 6). Die meisten intraoperativen Frakturen gehören zum Typ IC [14]. In diesen Fällen sehen wir die Indikation zum Anbringen von Cerclagen oder empfehlen die Zugschraubenosteosynthese. Frakturen des Typs IV machen weniger als $2 \%$ der Fälle aus [14] und können mit einfacher Zugschraubenosteosynthese der Tuberositas tibiae versorgt werden.

Nicht selten sind auch periprothetische Frakturen nach unikondylärer Schlittenprothese zu finden (Abb. 2 und 7) und werden in ihrer Inzidenz bis zu $5 \%$ angegeben [6]. Als Hauptursache wird ein zu weit nach distal geführter sagittaler 
Sägeschnitt der tibialen Komponente diskutiert $[19,38]$. In einer biomechanischen Vergleichsstudie konnte gezeigt werden, dass die offene Reposition und winkelstabile Plattenostesynthese der Zugschraubenosteosynthese in ihrer Stabilität signifikant überlegen ist, sodass die Autoren bei periprothetischen Frakturen nach unikondylärer Schlittenprothese die Plattenosteosynthese empfehlen [38].

\section{Literatur}

${ }^{1}$ Aaron RK, Scott $R$. Supracondylar fracture of the femur after total knee arthroplasty. Clin Orthop Relat Res 1987; 219: 136-139

2 Adigweme OO, Sassoon AA, Langford J et al. Periprosthetic patellar fractures. J Knee Surg 2013; 26: 313-317

${ }^{3}$ Aldrian S, Schuster R, Haas $N$ et al. Fixation of supracondylar femoral fractures following total knee arthroplasty: is there any difference comparing angular stable plate fixation versus rigid interlocking nail fixation? Arch Orthop Trauma Surg 2013; 133: 921-927

${ }^{4}$ Bae DK, Song SJ, Yoon KH et al. Periprosthetic supracondylar femoral fractures above tota knee arthroplasty: comparison of the locking and non-locking plating methods. Knee Surg Sports Traumatol Arthrosc 2014; 22: 26902697

Beals RK, Tower SS. Periprosthetic fractures of the femur. An analysis of 93 fractures. Clin Orthop Relat Res 1996; 327: 238-246

${ }^{6}$ Berger RA, Meneghini RM, Jacobs IJ et al. Results of unicompartmental knee arthroplasty at a minimum of ten years of follow-up. J Bone Joint Surg Am 2005; 87: 999-1006

7 Brooks P. Extensor mechanism ruptures. Orthopedics 2009; 32

8 Buttaro MA, Farfalli G, Paredes Nunez $M$ et al. Locking compression plate fixation of Vancouver type B1 periprosthetic femoral fractures. J Bone Joint Surg Am 2007; 89: $1964-$ 1969

9 Chen F. Mont MA, Bachner RS. Management of ipsilateral supracondylar femur fractures following total knee arthroplasty. J Arthroplasty 1994: 9: 521-526

10 Chen SH, Chiang MC, Hung CH et al. Finite element comparison of retrograde intramedullary nailing and locking plate fixation with without an intramedullary allograft for dista femur fracture following total knee arthroplasty. Knee 2014; 21: 224-231

11 Culp RW, Schmidt RG, Hanks G et al. Supracondylar fracture of the femur following prosthetic knee arthroplasty. Clin Orthop Relat Res 1987; 222: 212-222

12 Ebraheim NA, Sochacki KR, Liu X et al. Locking plate fixation of periprosthetic femur fractures with and without cerclage wires. Orthop Surg 2013; 5: 183-187

13 Ehlinger M, Adam P. Abane L et al. Treatment of periprosthetic femoral fractures of the knee. Knee Surg Sports Traumatol Arthrosc 2011; 19: 1473-1478

14 Felix NA, Stuart MJ, Hanssen AD. Periprosthetic fractures of the tibia associated with total knee arthroplasty. Clin Orthop Relat Res 1997; 345: 113-124

15 Fulkerson E, Tejwani N, Stuchin S et al. Management of periprosthetic femur fractures with a first generation locking plate. Injury 2007; 38: 965-972
${ }^{16}$ Gondalia V, Choi DH, Lee SC et al. Periprosthetic supracondylar femoral fractures following total knee arthroplasty: clinical comparison and related complications of the femur plate system and retrograde-inserted supracondylar nail. J Orthop Traumatol 2014; 15: 201-207

17 Gujarathi N, Putti AB, Abboud RJ et al. Risk of periprosthetic fracture after anterior femoral notching. Acta Orthop 2009; 80: 553-556

${ }^{18} \mathrm{Ha}$ CW, Shon OJ, Lim SW et al. Minimally invasive plate osteosynthesis for periprosthetic distal femoral fractures after total knee arthroplasty. Knee Surg Relat Res 2014; 26: 27-32

${ }^{19}$ Hanssen $A D$, Stuart MJ. Treatment of periprosthetic tibial fractures. Clin Orthop Relat Res 2000; 380: 91-98

${ }^{20}$ Harlow ML, Hofmann AA. Periprosthetic fractures. In: Scott WN, ed. The Knee. St. Louis, MO: Mosby-Year Book; 1994: 1405-1417

${ }^{21}$ Herrera DA, Kregor PJ, Cole PA et al. Treatment of acute distal femur fractures above a totalknee arthroplasty - systematic review of 415 cases (1981-2006). Acta Orthop 2008; 79: $22-27$

22 Keating EM, Haas G, Meding JB. Patella fracture after post total knee replacements. Clin Orthop Relat Res 2003; 416: 93-97

23 Kiluçoğlu OI, Akgül T, Sağlam Yet al. Comparison of locked plating and intramedullary nailing for periprosthetic supracondylar femur fractures after knee arthroplasty. Acta Orthop Belg 2013; 79: 417-421

${ }^{24}$ Kim KI, Egol KA, Hozack WJ et al. Periprosthetic fractures after total knee arthroplasties. Clin Orthop Relat Res 2006; 446: 167-175

${ }^{25}$ Lachiewicz PF. Periprosthetic fracture between a constrained total knee arthroplasty and a long-stem total hip arthroplasty: treatment with a novel device. J Arthroplasty 2007: 22: 449-452

${ }^{26}$ Lee SS, Lim SJ, Moon YW et al. Outcomes of long retrograde intramedullary nailing for periprosthetic supracondylar femoral fractures following total knee arthroplasty. Arch Orthop Trauma Surg 2014; 134: 47-52

${ }^{27}$ Meding JB, Fish MD, Berend ME et al. Predicting patellar failure after total knee arthroplasty. Clin Orthop Relat Res 2008; 466: 2769-2774

${ }^{28}$ Meneghini RM, Keyes BJ, Reddy KK et al. Modern retrograde intramedullary nails versus periarticular locked plates for supracondylar femur fractures after total knee arthroplasty. J Arthroplasty 2014; 29: 1478-1481

29 Ortiguera CJ, Berry DJ. Patella fracture after total knee aerthroplasty. J Bone Joint Surg Am 2002; 84: 532-540

30 Pekmezci M, McDonald E, Buckley J et al. Retrograde intramedullary nails with distal screws locked to the nail have higher fatigue strength than locking plates in the treatment of supracondylar femoral fractures: a cadaverbased laboratory investigation. Bone Joint J 2014; 96-B: 114-121

31 Reuben JD, McDonald CL, Woodard PL et al. Effect of patella thickness on patella strain following total knee arthro- plasty. J Arthroplasty 1991; 6: 251-258

32 Ritter MA, Thong AE, Keating EM et al. The effect of femoral notching during total knee arthroplasty on the prevalence of postoperative femoral fractures and on clinical outcome. J Bone Joint Surg Am 2005; 87: 2411-2414

33 Rorabeck CH, Taylor JW. Classification of periprosthetic fractures complicating total knee arthroplasty. Orthop Clin N Am 1999; 30: 209-214

${ }^{34}$ Ruchholtz S, Tomás J, Gebhard F et al. Periprosthetic fractures around the knee - the best way of treatment. Eur Orthop Traumatol 2013; 4: 93-102

${ }^{35}$ Rupprecht M, Schlickewei C, Fensky F et al. [Periprosthetic and interimplant femoral fractures: Biomechanical analysis.] Unfallchirurg 2014 Jun 5 [Epub ahead of print]

${ }^{36}$ Schüttrumpf JP, Behzadi C, Balcarek P et al. Radiologically hyperdense zones of the patella seem to be partial osteonecroses subsequent to fracture treatment. J Knee Surg 2013; 26: 319-326

37 Scuderi G, Scharf SG, Maltzer LP et al. The relationship of lateral releases to patella viability in total knee arthroplasty. J Arthroplasty 1987; 2: 209-214

${ }^{38}$ Seeger JB, Jaeger S, Röhner E et al. Treatment of periprosthetic tibial plateau fractures in unicompartmental knee arthroplasty: plates versus cannulated screws. Arch Orthop Trauma Surg 2013; 133: 253-257

${ }^{39}$ Seo JG, Moon YW, Park SH et al. A case-control study of spontaneous patellar fractures following primary total knee replacement J Bone Joint Surg Br 2012; 94: 908-913

40 Singh JA, Jensen M, Lewallen D. Predictors of periprosthetic fracture after total knee replacement. An analysis of 21, 723 cases. Acta Orthopaedica 2013; 84: 170-177

${ }^{41}$ Sun ZH, Liu YJ, Li H. Femoral Stress and Strain Changes post-Hip, -Knee and -Ipsilateral Hip/ Knee Arthroplasties: a Finite Element Analysis. Orthop Surg 2014; 6: 137-144

42 Thelen S, Schneppendahl J, Jopen E et al. Biomechanical cadaver testing of a fixed-angle plate in comparison to tension wiring and screw fixation in transverse patella fractures. Injury 2012; 43: 1290-1295

43 Vallier HA, Immler W. Comparison of the 95degree angled blade plate and the locking condylar plate for the treatment of distal femoral fractures. J Orthop Trauma 2012; 26 : 327-332

${ }^{44}$ Wick M, Muller EJ, Muhr G. Supracondylar femoral fractures in knee endoprostheses: stabilizing with retrograde interlocking nail. Unfallchirurg 2001; 104: 410-413

${ }^{45}$ Xue H, Tu Y, Cai M et al. Locking compression plate and cerclage bond for type B1 periprosthetic femoral fractures. J Arthroplasty 2011; 26: 467-471

46 Zalzal P. Backstein D, Gross AE et al. Notching of the anterior femoral cortex during total knee arthroplasty characteristics that increase local stresses. J Arthroplasty 2006; 21: 737-743

Prof. Dr. med. Karl-Heinz Frosch Leiter Chirurgisch-Traumatologisches Zentrum

Chefarzt Abteilung Unfall- und Wiederherstellungschirurgie, mit Sektion Knie- und Schulterchirurgie, Sporttraumatologie Dr. med. Jürgen Madert Chirurgisch-Traumatologisches Zentrum

Leitender Oberarzt Abteilung Unfallund Wiederherstellungschirurgie

Asklepios Klinik St. Georg Lohmühlenstraße 5 20099 Hamburg www.unfallchirurgie-hamburg.com

k.frosch@asklepios.com 